\title{
The Self-inspections (Internal Audits) Process as a Part of the Pharmaceutical Quality System: Formation of a Risk-Based Approach to Internal Audits Planning
}

\author{
Valeriy Nikityuk ${ }^{1}$, Tetiana Karamavrova ${ }^{2}$ and V. Lebedynets ${ }^{2}$ \\ 1. SCC "VADEMECUM" Ltd, Kyiv 02140, Ukraine \\ 2. National University of Pharmacy, Kharkiv 61002, Ukraine
}

\begin{abstract}
The quality system of any modern pharmaceutical company is the pharmaceutical quality system (PQS), which extends the GMP standards to all stages of the medicinal productslife cycle, from pharmaceutical development to its withdrawal from production. The principal difference between PQS and GxP rules from other quality systems is that the medicinal product, its safety and efficacy is put at the forefront. At the same time, PQS implies a process approach to all components that should be aimed at achieving the main goal—ensuring and guaranteeing the quality of the medicinal product for the end user (patient) — and should be based on the quality risk management system. An integral part of PQS, as well as the GxP rules adopted in the European Union and PIC/S, is a process for self-inspections and/or quality audits, which regularly appraises the effectiveness and applicability of the PQS. This publication is dedicated to the definition of self-inspections (internal audits) as one of the PQS's processes. The article defines the main standard stages and develops a model of a risk-based approach to the self-inspections' planning in relation to processes of the pharmaceutical quality system.
\end{abstract}

Key words: Medicinal products, PQS, Self-inspection, internal audit, quality risk management, risk-based approach.

\section{Introduction}

The quality system of a modern pharmaceutical company (both manufacturers and distributors of medicinal products) is the Pharmaceutical Quality System (PQS), the requirements for which are defined in the International Conference on Harmonization of Technical Requirements for Registration of Pharmaceuticals for Humane Use (ICH) Q10 [1], as well as the Good Manufacturing Practices (GMP) rules $[2,3]$ and the Good Distribution Practices (GDP) rules $[4,5]$.

One of the mandatory components of the PQS, as well as of the GMP and GDP rules, adopted in the European Union (EU) and established by the International Pharmaceutical Inspection Cooperation/Scheme (PIC/S), is the process of

Corresponding author: Valeriy Nikityuk, $\mathrm{PhD}$, research field: pharmaceutical science. self-inspections and/or quality audits, which, like all other processes, should be applied to all stages of the pharmaceutical products life cycle, from Pharmaceutical Development, including Technology Transfer and Commercial Manufacturing, to Product Discontinuation.

The GMP and GDP rules themselves, and the provisions of PQS [1-5] establish only the basic principles of the self-inspections system but do not contain relevant detailed guidelines on procedures. Some recommendations regarding the audits process can be found in certain international standards (in particular, in ISO 19011) [6], which do not involve specialized standards in their turn and do not define the necessary processes for the pharmaceutical scope.

Moreover, self-inspections procedures are not subject to mandatory adequate assessment by regulatory authorities which conduct official inspections of pharmaceutical companies. For example, 
the Compilation of the Community Procedures on Inspections and Exchange of Information EMA/572454/2014 contains the following statement: "the system for performing self-inspections in the company should be examined, although the reports themselves should not normally be read by the inspector" [7]. The GMP rules adopted in the United States of America [8] and the corresponding guidelines for FDA inspectors do not generally contain separate sections and requirements regarding the internal audits for pharmaceutical companies and their inspection assessment. At the same time, the process of internal audits is crucial for every pharmaceutical company in order to understand its own level of compliance with the requirements for the production or distribution of pharmaceuticals, and the level of quality assurance. Furthermore, internal audits help to achieve the goals of PQS: to identify and implement appropriate product quality improvements, as well as process improvements, variability reduction, innovations and PQS enhancements, thereby increasing the ability to fulfill quality needs consistently. Given the general recommendations regarding the organization and conducting of the process of internal audits, pharmaceutical companies are required to develop independent approaches to the regulation of relevant procedures.

Besides that, PQS (like any other modern quality system) requires that all processes be based on the Quality Risk Management (QRM) system, which is defined in the ICH Q9 Guide [9], which should also be taken into account in the process of internal audit.

In order to achieve this goal, each company has to:

(a) develop its own internal audit strategy as a PQS process;

(b) determine the tactics of its implementation by developing, implementing and applying the appropriate procedures, which should be in the form of written procedures (SOPs).

At the same time, both the strategy and tactics of the internal audit process should take into account the principles of QRM.

\section{Materials and Methods}

The purpose of our scientific and practical research was the formation of a strategy and a standardized approach to the process of self-inspections in a pharmaceutical company, aimed at conducting an internal audit of the PQS processes and based on GMP and GDP rules, PQS principles, Guidelines for auditing management systems (ISO 19011), and with due consideration of the Quality Risk Management system.

The main stages of the internal audit process are comparable to the stages of the external audit process. In turn, the procedures for planning, organizing and conducting an external audit conducted by pharmaceutical companies in relation to suppliers (i.e. suppliers of raw materials, bulk products, finished products) and consumers (i.e. distributors) are to a certain extent comparable to the main provisions of the requirements for planning, organization and conducting official inspections as contained in the PIC/S, EMA, US FDA guidelines. This also applies to the principles of risk-based inspection planning, which are defined in the relevant EMA guidelines (A Model for Risk-Based Planning for Inspections of Pharmaceutical Manufacturers) [7] and PIC/S recommendations [10]. Therefore, it seems appropriate to modify the approach that is recommended by international regulatory authorities to risk-based inspection planning so that it can be reasonably applied to risk-oriented planning of internal audits.

The main tasks for achieving this goal and forming a detailed and unified model of risk-based self-inspection planning that should be taken into account are as follows:

(a) Designate the start and finish of the internal audit process taking into account the requirements of a process specified in a written form and relevant procedures;

(b) Define the processes of the PQS as objects (subject) of internal audits (similarly, one can 
determine the list of departments and/or products, which can also be objects of self-inspections);

(c) Recommend an algorithm of actions that will help implement the procedure of planning of internal audits in the scheme of the process of quality risk management;

(d) Modify the algorithm of actions and the recommended scheme of risk-based inspection planning for practical use in planning the internal audits of a pharmaceutical company and

(e) Develop appropriate recommendations, including consideration of the requirements for self-inspections established by the GMP/GDP rules.

Of course, any company can develop its own strategy and tactics for the process of internal audits with regard to the specific features of building its own quality system.

\section{Results and Discussion}

The principles and general requirements for the self-inspections process (internal audits) are defined by certain sections of the GMP, GMP API, GDP, GDP API rules, other good practices adopted by the EU and PIC/S. The purpose of internal audits is the self-checking by pharmaceutical companies of their own compliance with the principles of PQS and the requirements of good practices. One of the key requirements is that internal audits should be conducted according to predetermined and detailed programs, that is, on a planned basis [2].

Internal audits can be classified according to the subject matter of the audit. Moreover, the procedures for preparing, conducting and evaluating results for each type of audit may have their own specifics. This should be taken into account when regulating them in appropriate written procedures (SOPs). An example of the classification of internal audits formulated by the authors is shown in Fig. 1.

The quality system adopted and officially recognized for pharmaceutical companies is the PQS, which involves determining the main processes that should be aimed at ensuring and guaranteeing the quality of medicinal products. Thus, it seems that the most acceptable and correct is the application of the process approach when conducting internal audits to verify compliance with the principles of the quality system of a pharmaceutical company. That is, the main objects of self-inspection should not be structural units (as in a functional audit), but the processes of the PQS. And the main type of applied internal audit should be the planned audit of processes.

Every pharmaceutical company is unique and has to define independently such processes based on the specific features of its business, specificity and nomenclature of medicinal products which are manufactured and distributed by the company, specificity and scale of business and business processes, number and qualification of personnel, uniqueness of the organization structure, business activities which the company carries out independently and which it gives for outsourcing, and other factors. Thus, the main processes of the quality system of a medicinal products distributor may, for example, include:

- management of procurement and delivery of goods process;

- inventory management and handling of medicinal products during the storage process;

- forming orders and selling products to consumers process;

- transportation and vehicle management process;

- product management in the "cold chain" process;

- quality control process (including procedures for authorizing sales of products by a responsible person);

- complaints, quality defects and potentially non-conforming products management process;

- building, facilities, equipment management process (including the management of measurement technology means, HVAC-systems, and others);

- outsourcing activities management process;

- waste management process (including disposal);

- personnel training management process;

- good personnel organization management process; 


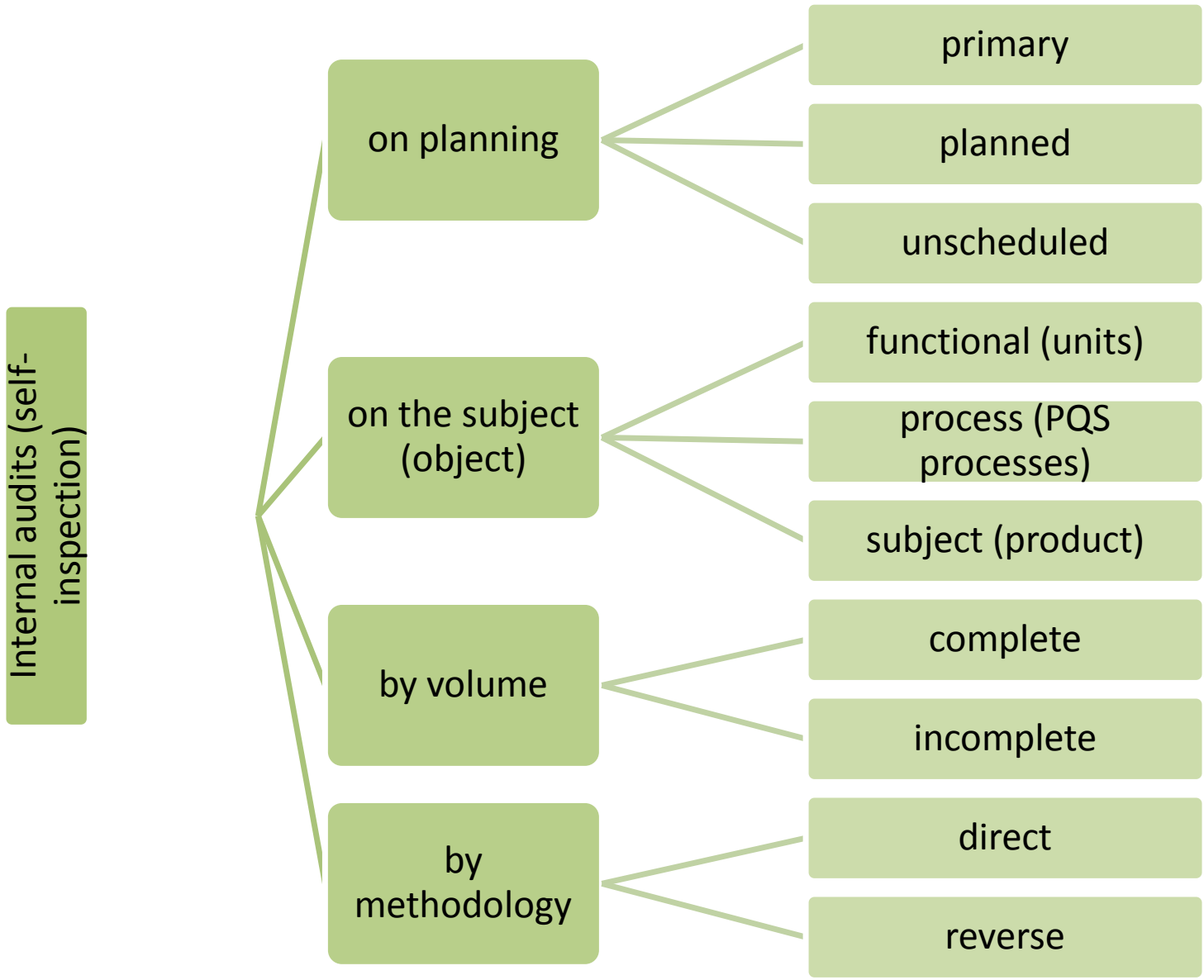

Fig. 1 An example of the self-inspections classification.

- good documentation management process (including management of records and data);

- quality risk management process;

- quality management process (including deviation control procedures, change control, CAPA, etc.);

- qualification and validation management process;

- the management review process;

- other processes.

The process of internal audits itself, in turn, is also one of the processes (or alternatively a sub-process) of the PQS. In order to ensure the proper functioning of this process as a whole and all its components, certain written procedures (SOPs) should be developed for each stage of the process considering the requirements of the management process of proper company documentation. At the same time, it seems relevant that the documentation system shall be formed and structured according to the pyramid principle with the definition of possible levels of documents and the principle of assigning documents to a particular level. An example of such a structure of the documentation system for the internal audit process is presented in Fig. 2.

Specific types of documentation for the self-inspections process are the following: the Guide (General SOP) on the process itself, prospective Schedule Plans/Self-Inspection Program (e.g., for a year), Plan/Program for each self-inspection and relevant protocols (checklists/primary records of observations, protocols of deviations), Report on each self-inspection, generalized (final) Review of all conducted self-inspections (in accordance to the prospective plan) with a review of the results and performance evaluation, as well as individual written practical methods (SOPs) for each individual procedure. 


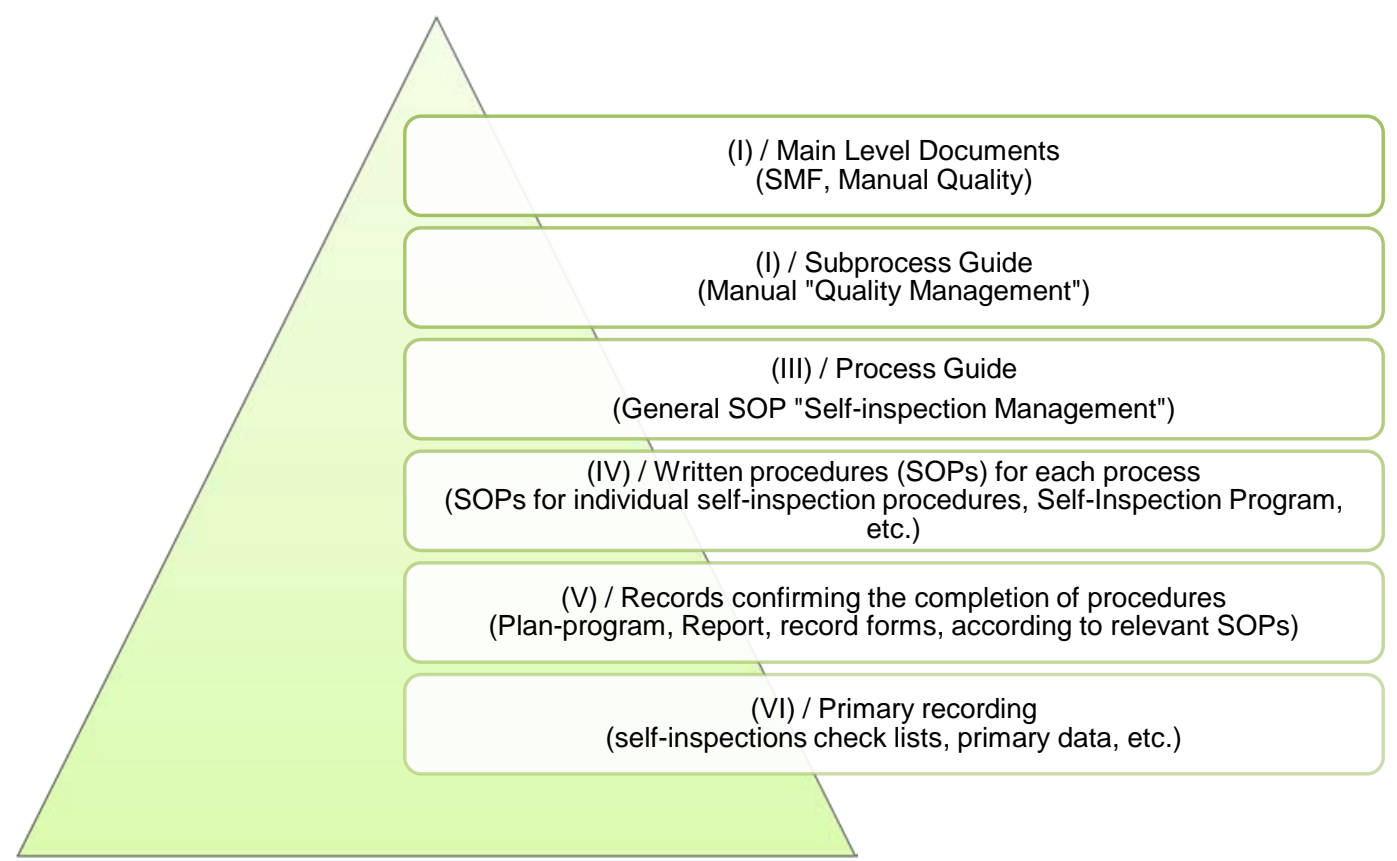

Fig. 2 An example of the structure of the good documentation system with the definition of possible levels of documents and the principle of assigning documents to a particular level using the example of the self-inspections (internal audits) process.

At the same time, the procedures of internal audits with regard to the requirements of modern approaches to the quality system should be based on risk analysis and take into account all factors influencing the effectiveness (efficiency) of the internal audit process. The format of the classical approach was modified by identifying the following "main factors" that may affect the quality and efficiency of the internal audit process: “men” ("personnel”), “methods” (“documentation”), “quality control”, “quality assurance”, “ambiance” (“environment”), "process”. Conventionally, this approach can be defined as “2M+2Q+A+P”. The authors carried out such an analysis using the above formula and the results are presented in Fig. 3 in the form of Ishikawa diagram.

As it was mentioned above, the basic self-inspection procedures should include planned audits of processes. Considering the fact that there may be quite a lot of the processes within the framework of PQS, and that the main resources for carrying out self-inspections (personnel and time) are quite limited in most pharmaceutical companies, the planned approach becomes critical for such companies. In addition, the practical experience of pharmaceutical companies shows that some processes in the framework of PQS "work" better than others, therefore, in practice, it is advised to "focus" self-inspection on more "problematic" processes. A risk-based process of planning internal audits can help in choosing the right focus of audits, their frequency and depth.

The PIC/S and EMA recommendations [7, 10] formulated for the planning of official inspections can be taken as the basis of the risk-based planning procedure for internal audits. The authors propose a modification of this approach to ensure the possibility of a unified application of risk-based planning for the process of internal audits. The general algorithm of the risk-oriented approach when planning internal audits is presented in the form of a diagram in Fig. 4. In forming this approach, we considered the model of the system of quality risk management, which is proposed by ICH [9]. The proposed risk-based approach scheme for planning internal audits, "embedded" in the general model of the quality risk management system, is presented in Fig. 5. 


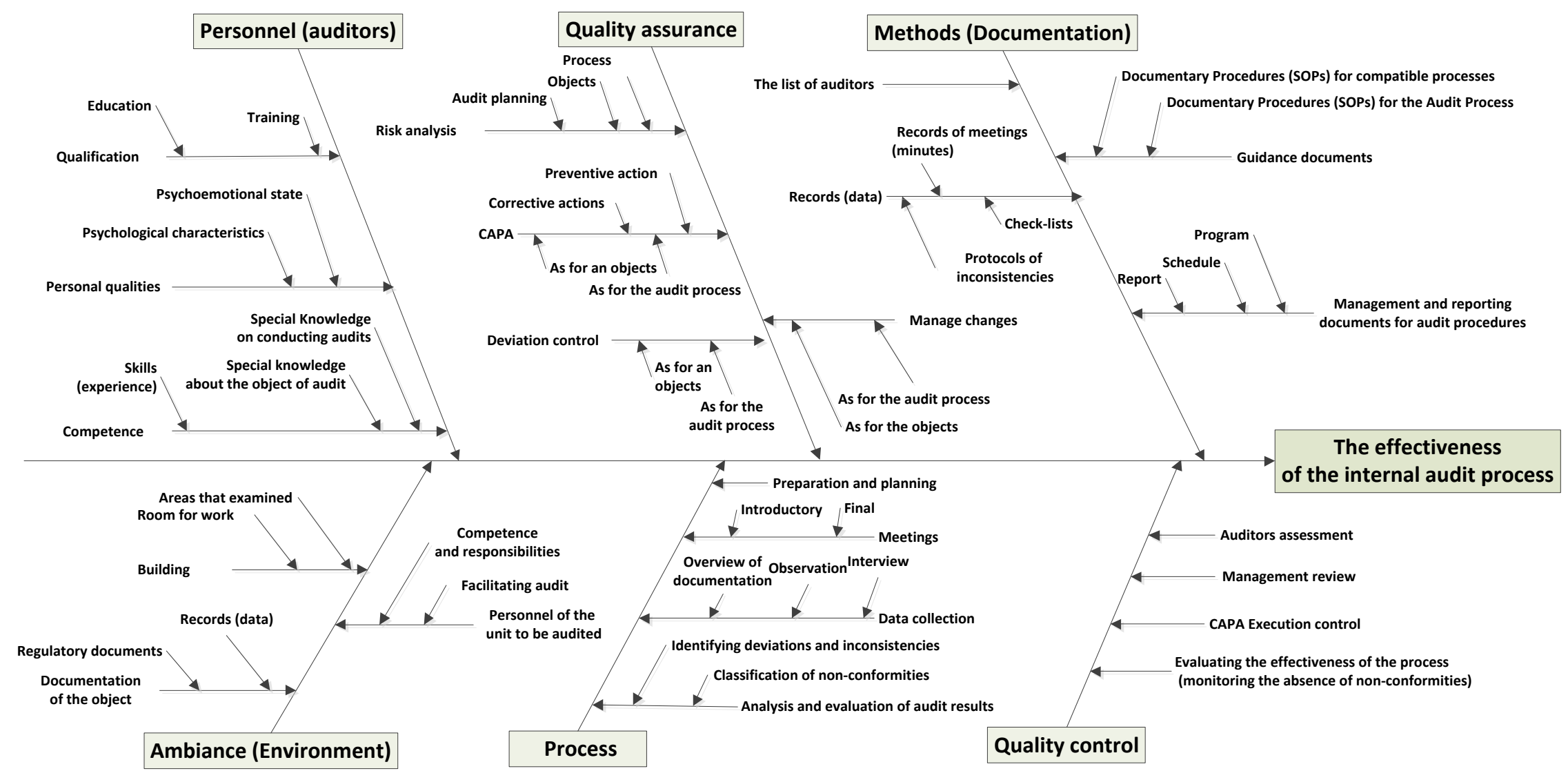

Fig. 3 An example diagram of determining factors influencing the quality and effectiveness of the self-inspections (internal audits) process in the format of a modified Ishikawa diagram. 
The Self-inspections (Internal Audits) Process as a Part of the Pharmaceutical Quality System: Formation of a Risk-Based Approach to Internal Audits Planning

1

- start of risk management process for risk-based self-inspection planning

- object selection (units/process/product);

- definition of a group of experts (analysts) for risk analysis

2

- risk identification

- the definition of permanent risk factors for the object;

- identifying variable risk factors for an object

3

- risk analysis

4

- risk evaluation

5

- risk reduction

- determination of recommendations on the frequency of audits;

- determination of volume requirements (time-consiming) for auditing;

- identifying recommendations for focus and audit depth;

- recommendations on the number of auditors (if necessary).

6

- risk acceptance

- definition of audit plan

- results

7

- approval (autorization) of the audit plan

8

- risk review (monitoring)

- conducting scheduled audits

Fig. 4 An algorithm of the risk-based approach procedure of planning internal audits. 
Formation of a Risk-Based Approach to Internal Audits Planning

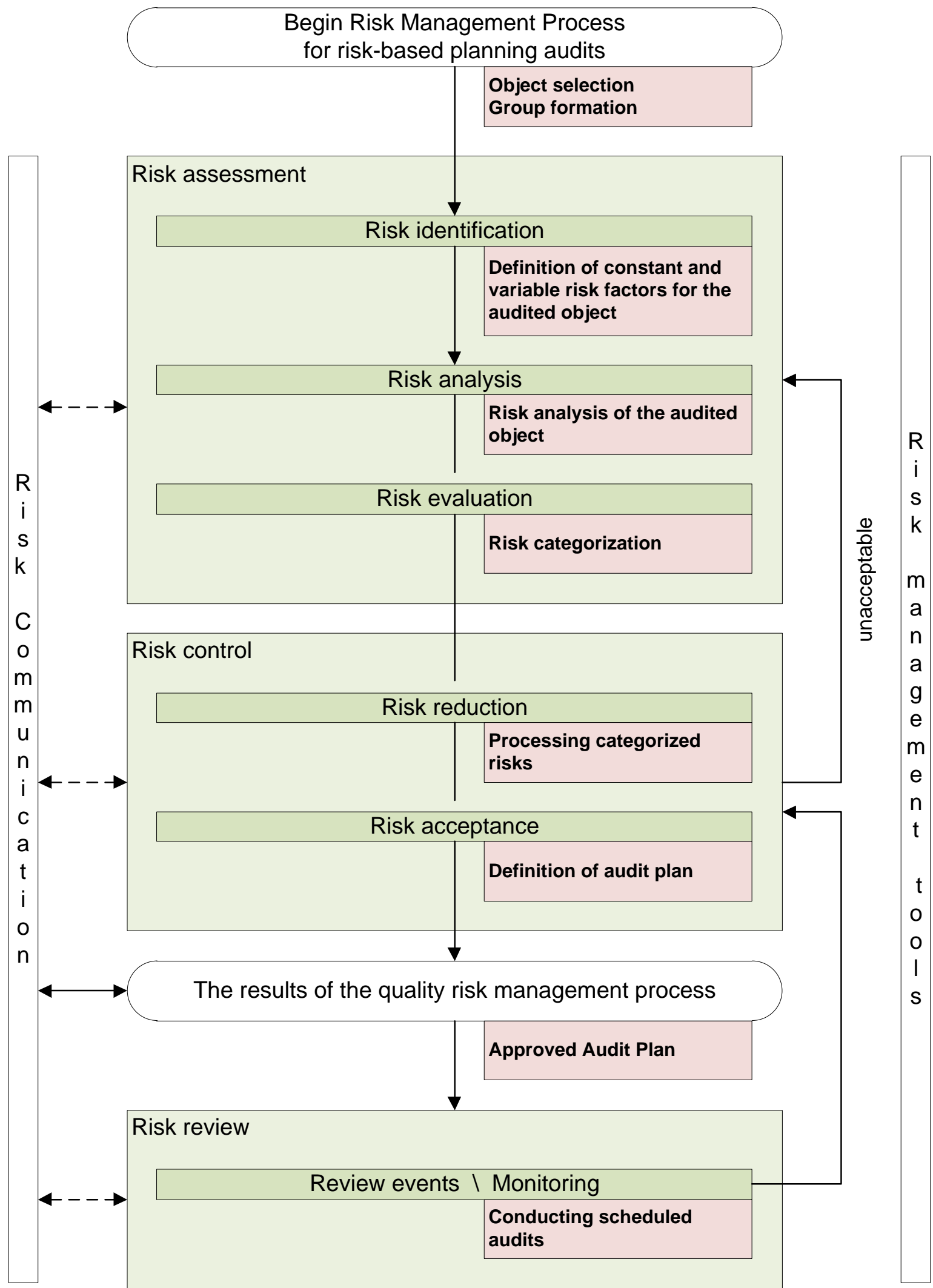

Fig. 5 A model of a risk-based approach to the planning of self-inspections (internal audits) based on a model of quality risk management. 
The proposed model is applicable for the organization of regular planned internal audits regardless of the subject/object (functional, process or subject audit), with the exception of the first audit with a goal, conducted for a specific object (division, process or product). In accordance with the proposed model, the procedure includes the following main steps:

$\checkmark$ Drawing up a risk analysis protocol based on the results of the audit (including the first audit with the goal);

\section{$\checkmark$ Risk classification;}

$\checkmark$ Identifying key recommendations in terms of frequency and resources for a subsequent planned audit;

$\checkmark$ Periodic review and updating of the plan in the light of new knowledge and improvements that arise after the generation of the plan.

The template of the risk analysis protocol includes 5 parts (I, II, III, IV, V).

Part I-the introductory part of the template, which is intended to define the object (subject) of the audit. It may be a specific division of the pharmaceutical company, a process of a PQS, or a product. Any other facility (for example, a separate production site or quality control laboratories, an office responsible for pharmacovigilance, etc.) can be defined, depending on certain goals and objectives that may be specific to each particular company.

Part II-contains a risk analysis, which includes determining the Intrinsic risk of the object and the risk of deviations from the established requirements for ensuring product quality, their violations or non-compliance.

The Intrinsic risk of the object (subject) of the audit should be characterized by three levels: level 1 (low risk), level 2 (medium risk) and level 3 (high risk). The company is allowed to determine a greater number of levels (for example, 4 or 5) for itself.

The Intrinsic risk of an object is defined as a constant value and is initially assigned to each object. To determine the complexity of an object which is a PQS process, the following characteristics can be taken into account:

$\checkmark$ Direct or indirect impact on product quality, including any impact during the shelf life;

$\checkmark$ The number of "associated" processes;

Applicability to product groups (for example, sterile or non-sterile, standard or non-standard processes for the production or handling of products, etc.);

$\checkmark$ Number of units/personnel involved;

$\checkmark$ The use of outsourcing (for example, the involvement of third-party companies in the implementation of individual stages of the process);

$\checkmark$ The presence or absence of risk analysis for the entire process;

$\checkmark$ Complexity or scope of documentation of the process;

$\checkmark$ The level of severity of changes that may be made to the process or related procedures;

$\checkmark$ Other factors.

If the object for internal risk is a process, then it can be determined on the basis of the matrix of ratios of each of the processes and their conditional characteristics, which are proposed by the authors. An example of such a matrix is presented in Table 1.

The determination of the risk of deviations from the established requirements for ensuring the quality of the product, their violations or non-compliance may be based on the number and classification of non-compliances revealed during the internal audit. The risk of deviations from the established requirements may also be divided into 3 levels, which can be described as follows:

$\checkmark$ Level 1-low risk level (absence of critical and major deficiencies according to the results of internal audit);

$\checkmark$ Level 2-average risk level (absence of critical deficiency major and the presence of no more than 5 major deficiencies according to the results of the internal audit);

$\checkmark$ Level 3-high risk level (the presence of critical or more than 5 major deficiencies). 
Table 1 An example of the matrix for determining the internal risk of certain processes of the PQS (for a distributor).

\begin{tabular}{|c|c|c|c|c|c|c|c|c|c|}
\hline \multirow{3}{*}{ Processes } & \multicolumn{7}{|c|}{ Process Characteristics } & \multirow{2}{*}{\multicolumn{2}{|c|}{$\begin{array}{c}\text { The Intrinsic risk of an } \\
\text { object (process) }\end{array}$}} \\
\hline & \multirow{2}{*}{$\begin{array}{l}\text { Impact on product } \\
\text { quality, including } \\
\text { during the shelf life }\end{array}$} & \multirow{2}{*}{$\begin{array}{c}\text { Number of } \\
\text { units/personnel } \\
\text { involved }\end{array}$} & \multirow{2}{*}{$\begin{array}{l}\text { Number of } \\
\text { "related" } \\
\text { processes }\end{array}$} & \multirow{2}{*}{$\begin{array}{c}\text { Complexity or scope } \\
\text { of documentation of } \\
\text { the process }\end{array}$} & \multirow{2}{*}{$\begin{array}{l}\text { The use of } \\
\text { works on } \\
\text { outsourcing }\end{array}$} & \multirow{2}{*}{$\begin{array}{c}\text { Presence or absence } \\
\text { of risk analysis for } \\
\text { the whole process }\end{array}$} & \multirow[b]{2}{*}{ Other ... } & & \\
\hline & & & & & & & & $\begin{array}{l}\text { Amount of } \\
\text { points }\end{array}$ & risk level \\
\hline $\begin{array}{c}\text { Acceptance, storage and } \\
\text { distribution of products } \\
\text { management }\end{array}$ & & & & & & & & & \\
\hline Quality control management & & & & & & & & & \\
\hline $\begin{array}{c}\text { Transport and transportation } \\
\text { management }\end{array}$ & & & & & & & & & \\
\hline $\begin{array}{l}\text { Handling products in the } \\
\text { "cold chain" }\end{array}$ & & & & & & & & & \\
\hline $\begin{array}{c}\text { Handling potentially } \\
\text { nonconforming product } \\
\text { management }\end{array}$ & & & & & & & & & \\
\hline \begin{tabular}{|l} 
Good documentation and \\
records (data) management
\end{tabular} & & & & & & & & & \\
\hline $\begin{array}{c}\text { Premises and equipment } \\
\text { management }\end{array}$ & & & & & & & & & \\
\hline $\begin{array}{c}\text { Outsourcing activities } \\
\text { management }\end{array}$ & & & & & & & & & \\
\hline$\ldots$ & & & & & & & & & \\
\hline
\end{tabular}


Table 2 An example of an object risk category (for self-inspections).

\begin{tabular}{llll}
\hline $\begin{array}{l}\text { Complexity } \\
\text { (comlince-related risk) }\end{array}$ & Low & Medium & High \\
\cline { 2 - 4 } Low & A (Low) & A (Low) & B (Medium) \\
Medium & A (Low) & B (Medium) & C (High) \\
High & B (Medium) & C (High) & C (High) \\
\hline
\end{tabular}

Table 3 An example of determining the frequency of planned self-inspections depending on the risk category.

\begin{tabular}{lll}
\hline \multirow{2}{*}{ Risk category } & \multicolumn{2}{c}{ The recommended frequency of planned audit } \\
\cline { 2 - 3 } & Definition & Periodicity \\
\hline A & Reduced frequency & 1 time in 2 years \\
$\mathrm{B}$ & Moderate frequency & Once a year \\
$\mathrm{C}$ & Increased frequency & At least 2 times a year \\
\hline
\end{tabular}

When identifying non-compliances that are detected during self-inspections, the authors applied their categorization in accordance with the $\mathrm{PIC} / \mathrm{S}$ recommendations [11], but each company can use a different approach or determine more risk levels (for example, 4 or 5).

Part III-determines the categorization of the object based on the conducted risk analysis (in accordance with Part II). At the same time, the risk category of an object is determined by the ratio of the Intrinsic risk of the object (subject), the audit of which is planned, and the level of risk of deviations from the specified requirements. Such a ratio of these two components can be presented in the form of a matrix, an example of which is given in Table 2 .

It is proposed to establish 3 categories of risk of an object:

$\checkmark$ A category-low risk level;

$\checkmark$ B category-medium risk level;

$\checkmark$ C category-high risk level.

Part IV-provides for the determination of recommendations regarding the frequency and scope of the subsequent planned audit.

The frequency of planned audits is determined depending on the risk category (calculated in accordance with Part III). For example, if the object belongs to the $\mathrm{C}$ category (high risk), the frequency of planned audits can be 1 time per quarter (or 1 time in half a year). When the risk belongs to the $\mathrm{B}$ category (medium), the frequency of planned self-inspections can be 1 time per year. If the A category is assigned to the risk (low risk level), the frequency of planned audits can be 1 time in 2 years or even less often. An example of the matrix for determining the frequency of audits is given in Table 3. It is understandable, that each company has the right to apply its own approach and determine a different frequency in accordance with the risk categories of the object.

In addition, when determining the scope of the subsequent planned audit, it is advisable to base on the following additional components:

$\checkmark$ The recommended focus and depth of the subsequent planned audit;

$\checkmark$ The recommended time for the next subsequent planned audit;

$\checkmark$ The need for special knowledge (competence) in its conduct.

For large companies that have separate divisions of internal auditors, one more component can be recommended, i.e. the required number of auditors, which should also be compared with the required audit time. For most companies that do not have such units in their organizational structure, the number of auditors is determined by their capabilities, but as for the number of auditors for each specific audit, it shall always be reasonable to appoint at least two auditors from the list of approved internal auditors.

These components are determined by the auditors based on the results of the conducted audit.

When determining the frequency of internal audits 
based on the risk category, as well as the recommended time for it, and determining the need to involve specialists with special knowledge, it is advisable to take into account additional factors that may be determined by the auditors based on the results of the conducted self-inspections. In particular:

$\checkmark$ Completeness of the audit-the conducted audit was complete (for example, in all procedures of the process and in all participating units) or incomplete. In the case of an incomplete audit, it is important to emphasize that during repeated planned self-inspection there is a need to check those components that have not been evaluated previously;

$\checkmark$ Planned changes in relation to the audit object, which, in the opinion of the auditors, may require attention during the subsequent planned self-inspection;

$\checkmark$ Activities of CAPA plans (for example, in relation to significant deviations or changes) that were not closed at the time of the audit, but the implementation of which should be taken into account during subsequent planned self-inspections;

$\checkmark$ Objects that were not subject to audit, but which are "associated" with it and which, considering the identified non-compliances, may potentially have non-compliances affecting the final quality of the product or the proper functioning of the PQS;

$\checkmark$ Any other issues and aspects that, in the opinion of the auditors who carried out the self-inspection, require attention during the subsequent planned audit.

Taking into account the analysis of these additional factors, the auditors can determine recommendations for increasing or decreasing man-days. For example, if an audit was incomplete, then the number of man-days for the next planned audit should be increased and vice versa.

The definition of such recommendations as in the previous examples can be based on the corresponding matrices, in which the basic conditions for each such factor can be formulated for increasing or decreasing man-days for the subsequent planned internal audit. To this end, it is important to determine the basic costs of human and time resources for conducting internal audits. For example, in the absence of conditions for increasing or decreasing the number of man-days, the internal base standard for small companies or for one pharmaceutical warehouse of a distributor company can be set as 2 man-days (implying self-inspection during 1 working day by two auditors). The standard amount of time to conduct an audit of each possible audit object can also be based on the determination of audit days, while the concept of an audit day can itself be determined, for example, as 0.5 man-days. Of course, the number of man-days or audit days for self-inspection of each object will be different and will depend on the complexity of the object, the amount of information that must be considered during the audit, the degree of influence on the goal of the audit (for example, on ensuring the quality of the product when it is production or distribution), a number of other factors.

Part V-the final part of the template, which contains the date of registration of the protocol and the signatures of the auditors who conducted the risk analysis.

\section{Conclusions}

Thus, we have proposed a model of the process of internal audits (self-inspections) as a process of a PQS with the consideration of the principles of the quality risk management system (in particular, we have applied an analysis of causal relationships of factors influencing the effectiveness and efficiency of the system of internal audits). An approach to risk-based planning for self-inspections for pharmaceutical companies was developed and formulated on the basis of a modification of the model recommended by international regulatory authorities for risk-based planning of official inspections. It seems that the proposed approaches can be used or modified for practical application by pharmaceutical companies in the process approach to the process of internal audits 


\section{Formation of a Risk-Based Approach to Internal Audits Planning}

and their risk-based planning.

\section{References}

[1] ICH Q10. 2008. Pharmaceutical Quality System.

[2] The Rules Governing Medicinal Products in the European Union. Volume 4. EU Guidelines to Good Manufacturing Practice Medicinal Products for Human and Veterinary Use.

[3] PE 009-14 Guidelines to Good Manufacturing Practice for Medicinal Products (1 July 2018).

[4] The Rules Governing Medicinal Products in the European Union. Volume 4. Guidelines on Good Distribution Practice of Medicinal Products for Human Use (2013/C 343/01).
[5] PE 011-01 PICIS Guidelines to Good Distribution Practice for Medicinal Products (1 June 2014).

[6] ISO/FDIS 19011:2018 Guidelines for auditing management systems.

[7] EMA/572454/2014. Compilation of Community Procedures on Inspection and Exchange of Information.

[8] Code of Federal Regulations Title 21. Part 211 Current Good Manufacturing Practice for Finished Pharmaceutical.

[9] ICH Q9. 2005. Quality Risk Management.

[10] PI 037. A Recommended Model of Risk-Based Inspection Planning in the GMP Environment (1 January 2012).

[11] PI 040. PIC\S Guidance on Classification of GMP Definition (1 January 2019). 